\title{
On Another Type of Transform Called Rangaig Transform
}

\author{
Norodin A. Rangaig*, Norhamida D. Minor, Grema Fe I. Pe nonal, \\ Jae Lord Dexter C. Filipinas, Vernie C. Convicto \\ Department of Physics, Mindanao State University-Main Campus, Marawi City 9700, Philippines \\ *Corresponding author: azis.norodinp6@gmail.com
}

\begin{abstract}
A new Integral Transform was introduced in this paper. Fundamental properties of this transform were derived and presented such as the convolution identity, and step Heaviside function. It is proven and tested to solve some basic linear-differential equations and had succesfully solved the Abel's Generalized equation and derived the Volterra Integral Equation of the second kind by means of Initial Value Problem. The Natural Logarithm (e.g $\log _{e} x=\ln x$ ) has been established and defined by means of modifying the Euler Definite Integral based on the Rangaig's fomulation. Hence, this transform may solve some different kind of integral and differential equations and it competes with other known transforms like Laplace, Sumudu and Elzaki Transform. Keywords: Rangaig Transform, Integral Transform, linear ordinary differential function, Integro-differential equation, Convolution Theorem.
\end{abstract}

Keywords: rangaig transform, integral transform, linear ordinary dierential function, integro-dierential equation, convolution theorem

Cite This Article: Norodin A. Rangaig, Norhamida D. Minor, Grema Fe I. Pe nonal, Jae Lord Dexter C. Filipinas, and Vernie C. Convicto, "On Another Type of Transform Called Rangaig Transform.” International Journal of Partial Differential Equations and Applications, vol. 5, no. 1 (2017): 42-48. doi: 10.12691/ijpdea-5-1-6.

\section{Introduction}

One of the most effective tools for solving problems in physics and engineering is using the transform method to obtain a solution for a given partial differential equations or ordinary differential equation by means of inverse transformation. Among these Transforms are the Fourier [1], Laplace [2], Hankel [3], Mellin [3] Sumudu [4], and Elzaki [5]. These transforms play a versatile role in solving many problems of physical interests which can be described by integro-differential equations with a given appropriate initial and boundary conditions. In these past years, applicability of these transforms were shown and considered by many authors $[6,7,8,9,10]$.

In this paper, we investigate a new transform which is also based on the previous transforms but in another domain. This Transform takes another form in process of transforming an equation and obtains the solution of the equation wherein it follows the set of functions $H$, an exponential order defined as

$$
H=\left\{\begin{array}{l}
h(t): \exists N, \lambda_{1}, \lambda_{2}>0,|h(t)|>N e^{\lambda_{i}|t|}, \\
t \in(-1)^{i-1} \times(-\infty, 0]
\end{array}\right\} .
$$

For the set in (1), the arbitrary constant $N$ must be a finite and the constants $\lambda_{1}, \lambda_{2}$ can be infinite or infinitely finite. Introducing a new transform which is defined in (1),

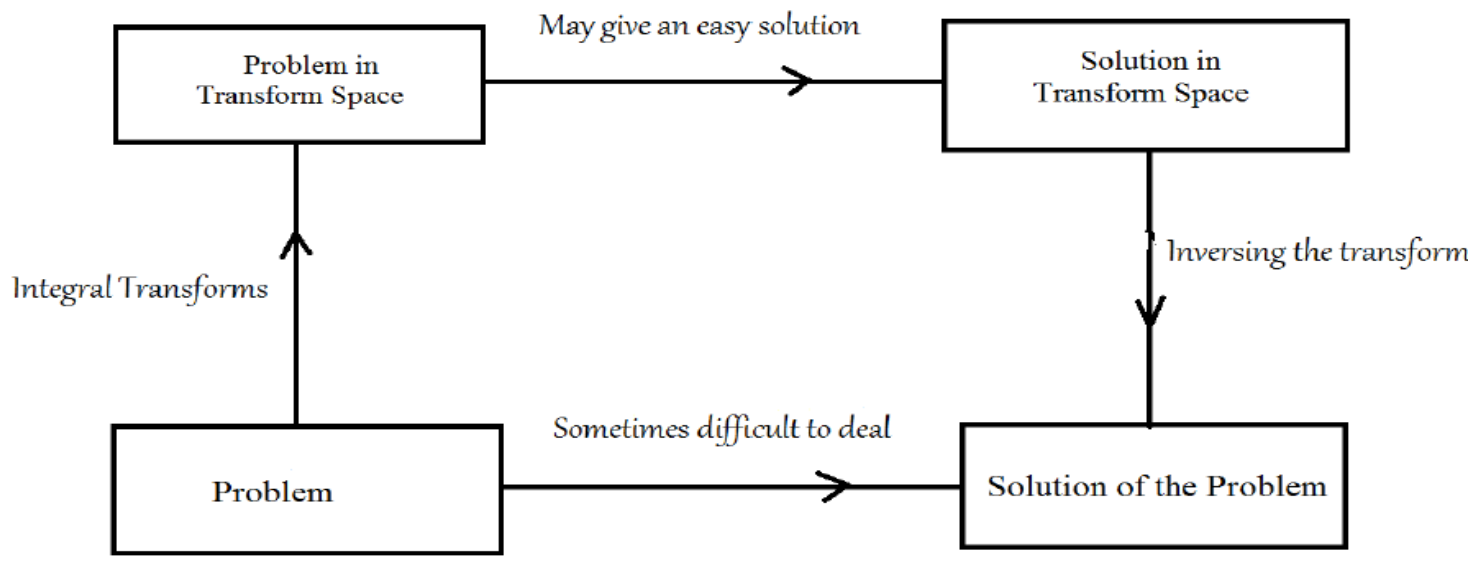

Figure 1. Schematic diagram when dealing with the solution of a problem using integral transform 


$$
\begin{aligned}
& \eta[h(t)]=\Lambda(\mu)=\frac{1}{\mu} \int_{-\infty}^{0} \exp (\mu t) h(t) d t, \\
& \frac{1}{\lambda_{1}} \leq \mu \leq \frac{1}{\lambda_{2}}
\end{aligned}
$$

called Rangaig Transform. In this transform, the variable $\mu$ factorizes the variable $t$ of the function $h$ or in another sense, the function $h(t)$ is mapped into the function $\Lambda(\mu)$ of $\mu$-space. Applicability and efficiency of this interesting transform will be shown in solving linear differential equations such as some problems on Classical Physics.

\section{Rangaig Transform of Some Functions}

For any function on $h(t) \in H$ such that the function $h(t)$ exists. Then the Rangaig Transform (2) is satisfied if the condition $t \geq 0$ for a function $h(t)$ and is piecewise continuous with decreasing exponential order. Properties of this transform are presented in the subsequent sections.

Definition 2.1. Let $\Lambda(\mu)$ denotes the Rangaig Transform of $h(t) \in H$. Then the following theorems holds:

Theorem 2.2. If $h(t)=1$, then its Rangaig Transform yields

$$
\eta[1]=\frac{1}{\mu} \int_{-\infty}^{0} \exp (\mu t) d t=\frac{1}{\mu^{2}}
$$

and for $h(t)=t$ yields

$$
\eta[t]=\frac{1}{\mu} \int_{-\infty}^{0} \exp (\mu t) t d t=-\frac{1}{\mu^{3}} .
$$

Now, the general form for $h(t)=t^{n}$, we have

$$
\eta\left[t^{n}\right]=\frac{1}{\mu} \int_{-\infty}^{0} \exp (\mu t) t^{n} d t=\frac{(-1)^{n} n !}{\mu^{n+2}} .
$$

Proof: Now, from the definition of the Rangaig Transform, we have

$$
\begin{aligned}
& \eta\left[t^{n}\right]=\frac{1}{\mu} \int_{-\infty}^{0} \exp (\mu t) t^{n} \\
& =\frac{1}{\mu}\left(\left[\frac{t^{n} \exp (\mu t)}{\mu}\right]_{-\infty}^{0}-\frac{n}{\mu} \int_{-\infty}^{0} \exp (\mu t) t^{n-1} d t\right) \\
& =\left[\frac{\exp (\mu t)}{\mu^{2}}\left(t^{n}-\frac{t^{n-1} n}{\mu}+\frac{n(n-1) t^{n-2}}{\mu^{2}}-\ldots \frac{(-1)^{n} n !}{\mu^{n}}\right)\right]_{-\infty}^{0} \\
& =\frac{(-1)^{n} n !}{\mu^{n+2}} .
\end{aligned}
$$

Theorem 2.3. For exponential function of the form $\exp (a t)$, then

$$
\eta[\exp (a t)]=\frac{1}{\mu} \int_{-\infty}^{0} \exp (\mu t) \exp (a t) d t=\frac{1}{\mu(\mu+a)} .
$$

Theorem 2.4. The Rangaig Transform of the trigonometric function such as $\cos (t)$ and $\sin (t)$ yields

$$
\eta[\sin (t)]=-\frac{1}{\mu}\left(\frac{1}{\mu^{2}+1}\right)
$$

and

$$
\eta[\cos (t)]=\frac{1}{\mu^{2}+1}
$$

respectively.

Proof: We can write the trigonometric function in terms of Taylor's series expansion

$$
\sin (t)=\sum_{n \geq 0} \frac{(-1)^{n} t^{2 n+1}}{(2 n+1) !}
$$

Then, obtaining the Rangaig Transform of $\sin (t)$ yields

$$
\eta[\sin (t)]=\frac{1}{\mu} \sum_{n \geq 0} \frac{(-1)^{n}}{(2 n+1) !} \int_{-\infty}^{0} \exp (\mu t) t^{2 n+1} d t
$$

and applying Theorem 2.2 and further simplification yields

$$
\eta[\sin (t)]=-\frac{1}{\mu^{3}} \sum_{n \geq 0}\left(\frac{-1}{\mu^{2}}\right)^{n} .
$$

Now, imposing the Binomial series expansion will result to

$$
\eta[\sin (t)]=-\frac{1}{\mu}\left(\frac{1}{\mu^{2}+1}\right)
$$

Similarly, for $\eta[\cos (t)]=\frac{1}{\mu^{2+1}}$.

Definition 2.5. If the function $h(t) \in H$ exists such that its $n$-th derivative also exists in the set (1), then let the Rangaig Transform of the $n$-th derivative of $h(t)$ be denoted as ${ }_{n} \Lambda(\mu)$ holds the next theorem.

Theorem 2.6. (Rangaig Transform for derivatives) If $h(t), h^{1}(t), \ldots, h^{(n)}(t) \in H$, then

$$
\begin{aligned}
& \eta\left[h^{(n)}(t)\right]={ }_{n} \Lambda(\mu) \\
& =(-1)^{n} \mu^{n} \Lambda(\mu)+(-1)^{n+1} \sum_{k=0}^{n-1}(-1)^{k} \mu^{n-2-k} h^{(k)}(0)
\end{aligned}
$$

Proof: (By Mathematical Induction). We verify for any $n \geq 0$ satisfies the theorem. So, for $n=0$, $\eta[h(t)]=\Lambda(\mu)$. For $\mathrm{n}=1$, we have

$$
\eta\left[h^{1}(t)\right]=\frac{1}{\mu} \int_{-\infty}^{0} \exp (\mu t) h^{1}(t) d t,
$$

applying integration by parts yields 


$$
\eta\left[h^{1}(t)\right]=-\mu \Lambda(\mu)+\frac{1}{\mu} h(0) .
$$

Suppose, there exist an integer $j$ such that $\eta\left[h^{(n)}(t)\right]$ holds for $j \geq n$. Letting $r(t)=h^{j}(t) \quad$ so $\quad$ that $r^{1}(t)=h^{j+1}(t)$. Hence,

$$
\begin{aligned}
\eta & {\left[h^{(j+1)}(t)\right]=\eta\left[r^{1}(t)\right] } \\
= & -\mu \eta\left[r^{1}(t)\right]+\frac{1}{\mu} r(0) \\
= & (-1)^{j+1} \mu^{(j+1)} \eta[h(t)] \\
& +(-1)^{j+2} \sum_{i=0}^{j-1}(-1)^{i} \mu^{j-i-1} h^{i}(0)+\frac{1}{\mu} h^{1}(t) \\
= & (-1)^{j+1} \mu^{(j+1)} \eta[h(t)] \\
& +(-1)^{j+1} \sum_{i=0}^{j}(-1)^{i} \mu^{j-1-i} h^{i}(0) .
\end{aligned}
$$

This finishes the proof.

Definition 2.7. Let the functions $m(t)$ and $h(t) \in H$. Then the next Theorem holds the Rangaig Transform of Integrals denoted by ${ }^{n} \Lambda(\mu)$.

Theorem 2.8. (Rangaig Transform for Integrals) If

$$
m^{n}(t)=\int_{0}^{t_{1}} \int_{0}^{t_{2}} \int_{0}^{t_{3}} \cdots \int_{0}^{t_{n+1}} h(T)(d T)^{n}
$$

such that $m(t) \in H$. Then, the Rangaig Transform of $m^{n}(t)$ is defined as

$$
\eta\left[m^{n}(t)\right]=\left[{ }^{n} \Lambda(\mu)\right]=\left(\frac{-1}{\mu}\right)^{n} \Lambda(\mu) .
$$

Proof: (By Mathematical Induction). We need to verify that Theorem 2.8 is satisfied for $n \geq 0$. For $n=0$, we can obtain $m^{0}(t)=h(t)$. For $n=1$, we get

$$
\eta[m(t)]=\left[{ }^{1} \Lambda(\mu)\right]=-\frac{1}{\mu} \Lambda(\mu)
$$

(see Theorem 2.6 and refer to it). To complete the proof, suppose there exists an integer $j$ such that $j \geq n$, for $j=n$, it is verified that this is true. Then for $j \geq n$

$$
\begin{aligned}
& \eta\left[m^{j+1}(t)\right]=\left[{ }^{j+1} \Lambda(\mu)\right] \\
& =\left(\frac{-1}{\mu}\right)^{j+1} \Lambda(\mu) \\
& =\left(\frac{-1}{\mu}\right)\left(\frac{-1}{\mu}\right)^{j} \Lambda(\mu) .
\end{aligned}
$$

Hence, the Proof has been done by Mathematical Induction.

Note that for multiple Integrals or antiderivative of a function $h(\mathrm{t})$, we can write its equivalence as

$$
\begin{aligned}
& \int_{0}^{t_{1}} \int_{0}^{t_{2}} \int_{0}^{t_{3}} \cdots \int_{0}^{t_{n+1}} h(T)(d T)^{n} \\
& =\int_{0}^{t_{1}}\left(t_{n}-t_{n-1}\right) h\left(t_{n+1}\right) d t_{n+1} .
\end{aligned}
$$

Definition 2.9. Consider the functions $h(t)$ and $r(t) \in H$.

Then next Theorem holds the Rangaig Transform for the Convolution Identity.

Theorem 2.10. The Rangaig Transform for the Convolution Identity given by

$$
\left(h^{*} r\right)(t)=\int_{0}^{t} h(t-T) r(T) d T
$$

is defined as

$$
\eta\left[\left(h^{*} r\right)(t)\right]=-\mu \Lambda_{1}(\mu) \Lambda_{2}(\mu)
$$

where $\Lambda_{1}(\mu)$ and $\Lambda_{2}(\mu)$ is the Rangaig Transform for $h(t)$ and $r(t)$ respectively.

Proof: Multiplying the Rangaig Transform of the functions $h(t)$ and $r(t)$ and to avoid complication, we assume that the limits are finite and thus resulting to

$$
\begin{aligned}
& \Lambda_{1}(\mu) \Lambda_{2}(\mu) \\
& =\frac{1}{\mu^{2}} \lim _{a \rightarrow \infty} \int_{-a}^{0} \exp (\mu x) h(x) d x \int_{-a-x}^{0} \exp (\mu y) r(y) d y .
\end{aligned}
$$

This formulation is possible since the integrands are considered to decrease exponentially. For the limit $a \rightarrow \infty$, the integrands are very complicated to deal with, thus to reduce this complication, let us assume an integer $t$ and $T$ such that $x=T-t, \quad y=T$. To verify these assumptions, we make use of the Jacobian's Transformation for differentials

$$
d x d y=-\left[\begin{array}{ll}
\frac{\partial x}{\partial t} & \frac{\partial y}{\partial t} \\
\frac{\partial x}{\partial T} & \frac{\partial y}{\partial T}
\end{array}\right] d t d T
$$

or simply $d x d y=-d t d T$. Substituting this expression to the above equation yields

$$
\begin{aligned}
& \Lambda_{1}(\mu) \Lambda_{2}(\mu) \\
& =-\frac{1}{\mu^{2}} \lim _{a \rightarrow \infty} \int_{-a}^{0} \exp (\mu t) \int_{0}^{t} h(t-T) r(T) d T d t \\
& =-\mu \eta[h(t-T) r(T)] .
\end{aligned}
$$

Corollary 2.11. Let the functions $h_{n}(t)$ and $r_{n}(T)$ be in $H$. Then the Rangaig Transform of the nth antidifferential of the Convolution Identity is defined as

$$
\eta\left[\left(h^{*} r\right)^{n}(t)\right]=(-\mu)^{n} \Lambda_{1}(\mu) \Lambda_{2}(\mu) .
$$

Furthermore, for any integer $n \geq 1$, we have the more generalized Convolution Identity defined by its Rangaig Transform as

$$
\begin{aligned}
& \eta\left[\left(g_{1} * g_{2} * g_{3} * \ldots * g_{n}\right)(t)\right] \\
& =(-\mu)^{n-1} G_{1}(\mu) G_{2}(\mu) G_{3}(\mu) \ldots G_{n}(\mu) .
\end{aligned}
$$


Proof: The same procedure in Theorem 2.8 for the expressions in this Corollary. The key to this proof is the associability and symmetric property of the convolution operator. Hence, Theorem 2.8 is the implication of Corollary 2.11 .

Definition 2.12. The duality relation of the Rangaig Transform to Laplace Transform exists if the function $h(t) \in H$ exists such that the function $h(t)$ is considered to change itself $h(t) \rightarrow h(-t) \in H$.

Theorem 2.13. (Duality relation of Rangaig Transform and Laplace Transform) If $h(t)$ and $h(-t)$ exist over $H$, then the relation of Rangaig Transform and Laplace Transform is

$$
\Lambda(\mu)=\frac{1}{\mu} F(-\mu)
$$

where $F(\mu)$ is the Laplace Transform of $h(t)$.

Proof: The Laplace Transform of the function $h(t)$ is

$$
F(\mu)=\int_{0}^{+\infty} \exp (-\mu t) h(t) d t
$$

following the given ranges of $t$ in (1). $t \rightarrow-t$ may exist and thus modifying the Laplace Transform into

$$
F(-\mu)=\int_{-\infty}^{0} \exp (\mu t) h(-t) d t
$$

Therefore the duality relation is satisfied.

Theorem 2.14. Let $M(t-a)$ be a Heaviside step function. Then its Rangaig Transform takes the form

$$
\eta[M(t-a)]=\frac{1}{\mu^{2}} \exp (a t)
$$

Proof: From the definition of the Rangaig Transform (2), we have

$$
\begin{aligned}
& \eta[M(t-a)]=\frac{1}{\mu^{2}} \exp (a t) \\
& \eta[M(t-a)]=\frac{1}{\mu} \int_{-\infty}^{0} \exp (\mu t) M(t-a) d t \\
& =\frac{1}{\mu} \int_{-\infty}^{0} \exp (\mu t) d t \\
& =\frac{1}{\mu}\left\{\int_{-\infty}^{0} \exp (\mu t) d t+\int_{0}^{a} \exp (\mu t) d t\right\} \\
& =\frac{1}{\mu^{2}} \exp (a \mu) .
\end{aligned}
$$

One proof of this can be done by the duality relation of the Rangaig Transform and Laplace Transform (Theorem 2.13).

\section{Some Application of Rangaig Transform}

In this section, we will present an application of Rangaig Transform to some differential equations. The following examples are governed by linear differential equations with initial condition wherein Rangaig Transform is used in solving some initial value problems described by ordinary differential equations.

\section{Example 3.1.}

Consider the first-order linear differential equation of the form

$$
\frac{d f}{d t}+f(t)=g(t)
$$

where $g(t)$ is an input function.

Sol'n: Let the initial condition be $f(0)=1$ so that we can write the Rangaig Transform for the first-order derivative as

$$
\eta\left[\frac{d f(t)}{d t}\right]=-\mu \Lambda(\mu)+\frac{1}{\mu} .
$$

Obtaining Rangaig transform will yield

$$
-\mu \Lambda(\mu)+\frac{1}{\mu}+\Lambda(\mu)=\eta[g(t)] .
$$

Further manipulation, we can obtain the general solution as

$$
\Lambda(\mu)=\frac{1}{\mu(\mu-1)}-\frac{\eta[g(t)]}{\mu-1}
$$

and taking the inverse Rangaig Transform leads to the solution. For $g(t)=0$, we have the solution

$$
f(t)=\exp (-t) \text {. }
$$

\section{Example 3.2.}

For an RC-circuit, the potential on the capacitor for $t=0^{-}$is $v_{0}$. After a long time $\left(t=0^{+}\right)$, the potential on the capacitor follows the first-order linear differential equation of the form

$$
v^{\prime}(t)+\frac{v(t)}{R C}=0, v^{\prime}\left(0^{-}\right)=v_{0}
$$

from Theorem 2.6, we have

$$
-\mu \Lambda(\mu)+\frac{v_{0}}{\mu}+\frac{1}{R C} \Lambda(\mu)=0
$$

and evaluating yields

$$
\Lambda(\mu)=\frac{v_{0}}{\mu\left(\mu-\frac{1}{R C}\right)} .
$$

Inversing the Rangaig Transform (using Theorem 2.3) we have the potential for the capacitor for long time

$$
v(t)=v_{0} \exp \left(-\frac{t}{R C}\right) \text {. }
$$

\section{Example 3.3.}

A bullet moving horizontally and experiences a resistive force during the motion. Then consider the equation of motion with constant $k$ [10]

$$
m \frac{d V(t)}{d t}=-k m V(t)
$$


then find the velocity at any time $\mathrm{t}$ and given that $V(0)=V_{0}$.

Sol'n: This problem is almost the same with the previous Example 3.2. Following similar step, we get the Rangaig Transformed equation of motion as

$$
\Lambda(\mu)=\frac{V_{0}}{\mu(\mu-k)}
$$

thus inversing, we obtain the solution

$$
V(t)=V_{0} \exp (-k t)
$$

\section{Example 3.4.}

Solve the differential equation

$$
\frac{d y}{d x}+2 y=x
$$

using the Rangaig transform if the initial condition is $y(0)=1$.

Sol'n: Again, Using the Theorem 2.6, we get the Rangaig Transform of the first derivative of the function as

$$
\eta\left[y^{\prime}\right]=-\mu \Lambda(\mu)+\frac{1}{\mu}
$$

thus the transformed differential equation takes the form

$$
-\mu \Lambda(\mu)+\frac{1}{\mu}+a \Lambda(\mu)=-\frac{1}{\mu^{3}} .
$$

Further manipulation, we get

$$
\begin{aligned}
& \Lambda(\mu)=\frac{1}{\mu(\mu-2)}+\frac{1}{\mu^{3}(\mu-2)} \\
& =\frac{5}{4}\left(\frac{1}{\mu(\mu-2)}-\frac{1}{4}\left(\frac{1}{\mu^{2}}\right)-\frac{1}{2}\left(\frac{1}{\mu^{3}}\right)\right) .
\end{aligned}
$$

Using the Previous theorems, we get the solution as

$$
y(x)=\frac{5}{4} \exp (-2 x)+\frac{1}{2} x-\frac{1}{4} .
$$

Example 3.5. (Application of Rangaig Transform on Abel's Generalized equation)

The Generalized Abel's equation is given by

$$
f(t)=\int_{0}^{t}(t-T)^{-\rho} r(T) d T, 0<\rho<1
$$

Where $f(t)$ is assumed to be known and $r(T)$ is unknown.

In this example, we will show the effectiveness of the Rangaig Transform in this kind of equation. Particularly, the validity of the Rangaig Transform on Convolution Theorem will be shown.

Taking the Rangaig Transform for both side of the equation yields

$$
n[f(t)]=\eta\left[\int_{0}^{t}(t-T)^{-\rho} r(T) d T\right] .
$$

Notice that the right-hand side of this expression demonstrates the Convolution Identity given in Theorem
2.10 if $h(t-T)=(t-T)^{-\rho}$. Then we can write the Rangaig Transform in accordance to the Convolution Theorem as

$$
n[f(t)]=\mu n\left[t^{-\rho}\right] n[r(t)]=-\mu\left\{\frac{(-1)^{-\rho}(-\rho) !}{\mu^{2-\rho}}\right\} n[r(t)] .
$$

Rearranging this expression yields

$$
\frac{1}{(-\rho) !}\left\{\frac{(-1)^{-\rho}}{\mu^{\rho}} \eta[f(t)]\right\}=-\frac{1}{\mu} \eta[r(t)]
$$

and multiplying the left side of the expression by $\frac{\mu(\rho-1) !}{\mu(\rho-1) !}$, we have

$$
\begin{gathered}
-\frac{\mu}{(\rho-1) !(\rho) !}\left\{\frac{(-1)^{\rho-1}(\rho-1) !}{\mu^{\rho+1}} \eta[f(t)]\right\}=-\frac{1}{\mu} \eta[r(t)] \\
-\frac{\mu}{(\rho-1) !(\rho) !}\left\{\eta\left[t^{\rho-1}\right] \eta[f(t)]\right\}=-\frac{1}{\mu} \eta[r(t)] .
\end{gathered}
$$

By using Theorem 2.10 and 2.8 we get

$$
\int_{0}^{t} r(T) d T=\frac{1}{(\rho-1) !(\rho) !} \int_{0}^{t}(t-T)^{\rho-1} f(T) d T
$$

then from [3], the factorial term of this expression can be written as

$$
(\rho) !(-\rho) !=\frac{\pi \rho}{\sin \pi \rho} .
$$

Substituting this expression to the previous equation and then differentiating. Hence, we have obtained the unknown term of Abel's equation as

$$
r(t)=\frac{\sin \pi \rho}{\pi} \frac{d}{d t} \int_{0}^{t} \frac{f(T)}{(t-T)^{1-\rho}} d T .
$$

Example 3.6. (Derivation of Volterra Integral Equation of the Second Kind Using Rangaig Transform)

Let us consider first an Initial Value Problem of the form

$$
h^{\prime \prime}(t)=r(t)+\sigma h(t)
$$

with the condition $h^{\prime}(t)=0, h(0)=1$. Now, let $h^{\prime \prime}(t)=f(t)$ then getting its Rangaig Transform yields

$$
\mu^{2} \eta[h(t)]-1=\eta[f(t)] .
$$

Simplifying, we get

$$
\eta[h(t)]=\frac{1}{\mu^{2}}+1 \frac{1}{\mu^{2}} \eta[f(t)] .
$$

Using Theorem 2.8 with $\mathrm{n}=2$, obtaining the inverse Rangaig Transform of this expression gives

$$
\begin{aligned}
& h(t)=1+\int_{0}^{t} \int_{0}^{t_{1}} f(T) d T \\
& =1+\int_{0}^{t}\left(t-t_{1}\right) f\left(t_{1}\right) d t_{1} .
\end{aligned}
$$


Therefore, we get the Volterra Integral Equation of the second kind as

$$
f(t)=r(t)+\sigma\left(1+\int_{0}^{t}(t-T) f(T) d T\right) .
$$

\section{Uniqueness of the Rangaig Transform}

The Gamma Function $\Gamma(n)$ is typically well defined for $n>0$, if $\mathrm{n}<0, \Gamma(n)= \pm \infty$. It is hard to define $\Gamma(n), n<0$ since it diverges for $n \rightarrow \infty$. The Gamma function can be defined by its second deffnition known as Euler's Definite Integral [13]

$$
\Gamma(n)=\int_{0}^{\infty} \exp (-t) t^{(n-1)} d t
$$

Logically, if the Gamma function happens to have a negative value of $n$, of course it is difficult to deal, but what we do is to create a counter part of the Gamma function just in case for $n$ to take values from negative. If we let $\Gamma(-n)$ be the counter form of the Gamma function if $n<0$. Another problem arises, that is, how can we make an equivalence form of $\Gamma(-n)$, and in order to do this, we need to modify the Euler Definite Integral based on the formulation of the Rangaig Transform. We have the proposed modification of the Euler Definite Integral based on the form of Rangaig Transform as

$$
\Gamma(-n)=(-1)^{(-n-1)} \int_{-\infty}^{0} \exp (t) t^{(-n-1)} d t, n<0 .
$$

The reason of this formulation is restraining the values of $\mathrm{n}$ to become negative. Since this formulation is based on Rangaig Transform, it is required that this result is applicable only to Rangaig Transform for $n<0$. Consider the Function $h(t)=t^{n}$ where $n<0$, then we have its Rangaig Transform as

$$
\eta\left[t^{n}\right]=(-1)^{(n+1)} \frac{\Gamma(-n)}{\mu^{n}}, n<0 .
$$

For which the Laplace Transform of $1 / t$ is $L\left\{\frac{1}{t}\right\}=\infty$. Hence, this gives us the property of a Natural Logarithm (Napierian).

Proposition 4.1. For a function $h(t)=\ln (t) \in H$. Then we have obtained the Rangaig Transform for Natural Logarithm defined as

$$
\eta[\ln |t|]=-\frac{1}{\mu} \eta\left[\frac{1}{t}\right], t \neq 0 .
$$

Proof: From the Integral definition of natural logarithm, we have

$$
\ln |t|=\int_{t}^{1}-d t
$$

Recall, from Theorem 2.8 the Rangaig transform of a given integral

$$
\eta\left[\int t^{n} d t\right]=\left(\frac{-1}{\mu}\right) \eta\left[t^{n}\right]
$$

for $n=-1$, we get

$$
\eta\left[\int_{t}^{1} d t\right]=\left(\frac{-1}{\mu}\right) \eta\left[\frac{1}{t}\right] .
$$

Actually, using the Uniqueness of Rangaig Transform, we have $\eta[\ln (\mp t)]= \pm 1$. Therefore, the Proposition is satisfied.

Lemma 4.2. For the function

$$
h(t)=\int t^{n} d t, n<0
$$

and

$$
r(t)=\frac{d}{d t}\left(t^{n}\right), n<0
$$

Then, thier Rangaig Transform takes the form

$$
\begin{aligned}
& \eta[h(t)]=\eta\left[\int t^{n} d t\right] \\
& =\frac{\Gamma(-n-1)}{\Gamma(-n)} \eta\left[t^{n+1}\right], n \leq-2, \\
& \eta[r(t)]=\eta\left[\frac{d}{d t}\left(t^{n}\right)\right] \\
& =\frac{\Gamma(-n+1)}{\Gamma(-n)} \eta\left[t^{n-1}\right], n \leq-1,
\end{aligned}
$$

respectively.

Proof: (By Mathematical Induction). We will verify that for $n \leq-2$ is true. For $n=-2$, we have

$$
\eta\left[\int t^{-2}\right]=\frac{\Gamma(1)}{\Gamma(2)} \eta\left[t^{-1}\right],
$$

which is true (can be verified by integrating). Suppose there exist an integer $j<0$ such that $|j| \geq|n|$, for $|j|=|n|$, it is indeed verified. Now, we will show that for all $|j|+1$ is true, that is

$$
\eta\left[\int t^{j+1}\right]=\frac{\Gamma(-j)}{\Gamma(-j+1)} \eta\left[t^{j}\right],|j| \geq|n| .
$$

This finishes the proof.

Similar proof can be done for

$$
\eta\left[\frac{d}{d t}\left(t^{n}\right)\right]=\frac{\Gamma(-n+1)}{\Gamma(-n)} \eta\left[t^{n-1}\right], n \leq-1 .
$$

Example 4.3. Effeciency of Uniqueness of the Rangaig Transform

In this following examples, we wil going to show the validity of the Uniqueness of the Rangaig Transform to some separable differential equation.

Consider a differential equation of the form

$$
(x+1) y^{\prime}=y^{2}
$$

where $y^{\prime}=\frac{d y}{d x}$. So, the equation can be immediately written as 


$$
\frac{d x}{1+x}=\frac{d y}{y^{2}} .
$$

Using Theorem 2.8 we have

$$
-\frac{1}{\mu} \eta\left[\frac{1}{1+x}\right]=-\frac{1}{\mu} \eta\left[\frac{1}{y^{2}}\right] .
$$

Now, Using the Uniqueness we get

$$
\eta[\ln (1+x)]=\mu
$$

and inversing both sides of the equation, we can obtain its solution as

$$
\ln (1+x)-\frac{1}{y}=0
$$

For general solution, we have

$$
\ln (1+x)-\frac{1}{y}=C, \text { where } C=\text { constant } .
$$

Example 4.4. Solve this differential equation

$$
(y+1) d x+x y d y=0
$$

by applying the Rangaig Transform.

Sol'n: Since the equation is separable we can write it as

$$
\frac{d x}{x}=-\frac{y d y}{y+1}=\left(\frac{1}{y+1}-1\right) d y .
$$

Applying the Rangaig Transform for both sides of the equation we have

$$
\begin{aligned}
& -\frac{1}{\mu} \eta\left[\frac{1}{x}\right]=-\frac{1}{\mu}\left\{\eta\left[\frac{1}{y+1}\right]-\eta[1]\right\} \\
& =-\frac{1}{\mu} \eta\left[\frac{1}{y+1}\right]+\frac{1}{\mu^{3}},
\end{aligned}
$$

and further manipulations, we have the solution as

$$
\ln (x)-\ln (y+1)+y=0 \text {. }
$$

\section{Conclusion}

The deffnition of Rangaig Transform and its application to the solution of ordinary differential equation has been presented. It was shown that the Rangaig Transform has a clearer and deeper connection to Laplace Transform. However, there are cases that Laplace Transform and Elzaki Transform cannot solve integro-differential equations but can be solved in Rangaig Transform. Therefore, the Rangaig Transform can be used as an effective tool in solving integro-differential equations.

\section{Acknowledgements}

The authors would like to thank the Department of Physics for the support and ideas in developing this paper. The authors would also like to thank the Mindanao State University-Main Campus for the support extended on this work.

\section{References}

[1] E. D. Rainvill and P. E. Bedient, Elementary Differential Equations, 6th ed., Macmillian Publishing Co., Inc., New York, pp. 170-232, 1981.

[2] L. Debnath, D. Bhatta, Integral Transform and thier Application, 2nd Edition; Chapman and Hall/CRC, 2006.

[3] G. B Arfken and H. J. Weber, Mathematical Methods for Physicists, 6th ed., Elsevier Inc., pp. 965-071, 2005.

[4] G. K. Watugala, Sumudu Transform- a new Integral Transform to Solve differential Equation and Controlling Enginnering, Math. Eng'g Induct., vol. 6, no.1, pp.319-329, 1998.

[5] T. M. Elzaki, The New Integral Transform Elzaki transform, Global Journal of Pure and AppliedMathematic, vol. 7, no. 1, pp.57-64, 2011.

[6] Zhang J., A Sumudu Based Algoritm for Solving Differential equations, Comp. Sci. J. Moldova, vol. 3, no. 15, pp.303-313, 2009

[7] T. Elzaki, S. Elzaki, and E. Elnour, On some applications of new integral transform 'Elzaki Transform', The Global Journal of Mathematical Sciences: Theory and Practical, vol. 4, no. 1, pp.15-23, 2012.

[8] S. Weera Koon, Application of Sumudu Transform to Partial Differential Equation. Int. J. Math. Educ. Sc. Tech., vol. 25, no. 2, pp. 277-283, 1994.

[9] T. M. Elzaki and S. M. Ezaki, On the connections between Laplace and ELzaki transforms, Adv. Theo. and Appl. Math., vol.67 no. 6, pp. 1-10. 2011

[10] T. M. Elzaki, S. M. Ezaki and E. M.A. Hilal, ELzaki and Sumudu transform for solving some differential equations Global Journal of Pure and Applied Mathematic, vol. 4, no. 8, pp. 167-173, 2012.

[11] M. R. Spiegel, S. Lipschutz, J. Liu, Mathematical Handbook and Formulas and Tables, Third Edition, McGraw-Hill Comp. 2009.

[12] S. T. Thornton ST, J. B. Marion JB, Classical Dynamics of Particles and Systems, Fifth Edition, Academic Press, 2004.

[13] G. Arfken, Mathematical Methods for Physicist, 4th edition; Academic Press, New York, (1985). 